\title{
Rotational broadening and conservation of angular momentum in post-extreme horizontal branch stars
}

\author{
G. Fontaine ${ }^{1}$ and M. Latour ${ }^{2}$ \\ ${ }^{1}$ Département de Physique, Université de Montréal, Succ. Centre-Ville, C.P. 6128, Montréal, QC, H3C 3J7, Canada \\ e-mail: fontaine@astro. umontreal.ca \\ ${ }^{2}$ Dr. Karl Remeis-Observatory \& ECAP, Astronomical Institute, Friedrich-Alexander University Erlangen-Nuremberg, \\ Sternwartstr. 7, 96049 Bamberg, Germany \\ e-mail: marilyn.latour@fau.de
}

Received 13 November 2017 / Accepted 29 December 2017

\begin{abstract}
We show that the recent realization that isolated post-extreme horizontal branch (post-EHB) stars are generally characterized by rotational broadening with values of $V_{\text {rot }} \sin i$ between 25 and $30 \mathrm{~km} \mathrm{~s}^{-1}$ can be explained as a natural consequence of the conservation of angular momentum from the previous He-core burning phase on the EHB. The progenitors of these evolved objects, the EHB stars, are known to be slow rotators with an average value of $V_{\text {rot }} \sin i$ of $\sim 7.7 \mathrm{~km} \mathrm{~s}^{-1}$. This implies significant spin-up between the EHB and postEHB phases. Using representative evolutionary models of hot subdwarf stars, we demonstrate that angular momentum conservation in uniformly rotating structures (rigid-body rotation) boosts that value of the projected equatorial rotation speed by a factor $\sim 3.6$ by the time the model has reached the region of the surface gravity-effective temperature plane where the newly-studied post-EHB objects are found. This is exactly what is needed to account for their observed atmospheric broadening. We note that the decrease of the moment of inertia causing the spin-up is mostly due to the redistribution of matter that produces more centrally-condensed structures in the post-EHB phase of evolution, not to the decrease of the radius per se.
\end{abstract}

Key words. stars: evolution - stars: rotation - stars: atmospheres - subdwarfs

\section{Astrophysical context}

The hot, hydrogen-rich subdwarf $\mathrm{O}(\mathrm{H}-\mathrm{sdO})$ stars are believed to be the direct descendants of the cooler He-core burning stars of spectral type sdB that populate the extreme horizontal branch stars (EHB). They correspond to the He-shell burning phase (usually referred to as the post-EHB phase) that immediately follows the EHB stage (Dorman et al. 1993; Han et al. 2003). They are found in a very wide range of effective temperature $\left(T_{\text {eff }}\right)$, from about $38000 \mathrm{~K}$ to upward of $80000 \mathrm{~K}$, and their surface gravities cover the interval $4.6 \lesssim$ $\log g \lesssim 6.4$ (see, e.g., Heber 2009, 2016). While the main atmospheric properties of these hot stars $\left(T_{\text {eff }}, \log g\right.$, and $\log$ $N(\mathrm{He}) / N(\mathrm{H})$ ) can generally be estimated from optical spectroscopy coupled to appropriate non-local thermodynamical equilibrium (NLTE) atmosphere models (Stroeer et al. 2007; Németh et al. 2012; Fontaine et al. 2014), very few of them have been characterized in terms of their chemical composition in detail. This is due both to the scarcity of metal lines in the optical spectra of sdO stars along with the inherently demanding task of computing NLTE models with metal line blanketing.

In this context, the recent study of Latour et al. (2018) has added four stars to the meager sample of three H-sdOs for which detailed abundance analyses using multiwavelength data have been carried out. The latter are AA Dor (Fleig et al. 2008; Klepp \& Rauch 2011), BD+284211 (Latour et al.
2013), and Feige 110 (Rauch et al. 2014). The additions of Latour et al. (2018) are Feige 34, Feige 67, AGK $+81^{\circ} 266$, and LS II $+18^{\circ} 9$, which have been found to show very similar abundance patterns and to be located very close to each other, around $T_{\text {eff }} \simeq 61400 \mathrm{~K}$ and $\log g \simeq 6.0$ (their average values) in the surface gravity-effective temperature plane. Among other results, and of particular interest here, all of these four stars have spectra that show the signature of rotational broadening with characteristic values of $V_{\text {rot }} \sin i$ between 25 and $30 \mathrm{~km} \mathrm{~s}^{-1}$. This adds significantly to the very few cases reported so far in which rotational broadening could be detected in $\mathrm{H}-$ sdOs - essentially on the basis of rare high-resolution UV observations.

For instance, Deleuil \& Viton (1992) were the first to report the need for a broadening mechanism with an amplitude of some 30 to $40 \mathrm{~km} \mathrm{~s}^{-1}$ in the IUE spectra of both LS II $+18^{\circ} 9$ and CPD $-71^{\circ} 172 \mathrm{~B}$. The latter is a near spectroscopic twin of the former, although $\mathrm{CPD}-71^{\circ} 172 \mathrm{~B}$ resides in a binary system with an F3-F4 main-sequence star (Viton et al. 1988). For their part, Becker \& Butler (1995) used a rotational velocity of $30 \mathrm{~km} \mathrm{~s}^{-1}$ to account for the profiles of the metal lines detected in the IUE spectrum of Feige 67. More recently, Ringat \& Rauch (2012) best reproduced the FUSE spectrum of the H-sdO star EC 11481-2303 ( $T_{\text {eff }}=55000 \pm$ $5000 \mathrm{~K}, \log g=5.8 \pm 0.3)$ by using a value of $V_{\text {rot }} \sin i$ $=30 \mathrm{~km} \mathrm{~s}^{-1}$ as well. Put together with the work of Latour et al. (2018), this suggests that rotational broadening of that 
order is likely a general characteristic of isolated post-EHB stars $^{1}$.

In comparison, it has been shown that the immediate progenitors of $\mathrm{H}$-sdOs, the EHB stars, are collectively much slower rotators. Indeed, in their important work on the rotational properties of sdB stars, Geier \& Heber (2012) have found that all the objects in their sample of 105 isolated EHB stars have values of $V_{\text {rot }} \sin i$ less than $10 \mathrm{~km} \mathrm{~s}^{-12}$. Their distribution of projected rotational velocities is consistent with a mean value of $7.7 \mathrm{~km} \mathrm{~s}^{-1}$ for EHB stars. This figure implies that there must be a significant spin-up during the post-EHB phase of evolution. As pointed out by Latour et al. (2018), in the absence of significant mass loss and accretion of matter, conservation of angular momentum in such isolated stars appears to be the most likely mechanism to account for this spin-up. Indeed, the $\mathrm{H}$-sdOs of interest here are more compact, with $\log g \simeq 6.0$, than a typical $\mathrm{sdB}$ progenitor, rather characterized by a value of $\log g \simeq 5.7$ (see, e.g., Heber 2009). This translates into a radius ratio of $R_{\mathrm{sdB}} / R_{\mathrm{H}-\mathrm{sdO}} \simeq 1.4$. For a uniform-density sphere rotating rigidly, it is well known from classical mechanics that the equatorial speed $V_{\text {rot }}$ scales as $1 / R$ if angular momentum is conserved. Hence, in this rough approximation of constant density, the $\mathrm{H}$-sdO would rotate some 1.4 times faster than the $\mathrm{sdB}$ progenitor. This falls quite short of the needed factor to account for the values of 25 to $30 \mathrm{~km} \mathrm{~s}^{-1}$, but, of course, real stars are far from being uniform-density bodies. Here, we reexamine the question of angular momentum conservation with the help of realistic evolutionary models.

\section{Conservation of angular momentum}

We start by recalling some basic concepts of classical mechanics. Consider a sphere rotating uniformly (like a rigid body) about its axis of symmetry. Its total angular momentum $L$ is given by

$L=I \omega=\frac{I V}{R}$,

where $I$ is the moment of inertia of the sphere with respect to its symmetry axis, $\omega$ is the angular velocity, $V$ is the equatorial speed, and $R$ is the radius. Assuming that angular momentum is conserved, and considering the "evolution" of the sphere from configuration \#1 to configuration \#2, we find that

$\frac{I_{1} V_{1}}{R_{1}}=\frac{I_{2} V_{2}}{R_{2}}$.

For a uniform-density sphere, it is well known from standard textbooks in classical mechanics that

$I=\frac{2}{5} M R^{2}$,

1 We note, in this context, that the rotational velocity of $V_{\text {rot }}=35 \pm 5 \mathrm{~km} \mathrm{~s}^{-1}$ (Fleig et al. 2008) or $30 \mathrm{~km} \mathrm{~s}^{-1}$ (Klepp \& Rauch 2011) inferred for the $\mathrm{H}$-sdO primary of the close binary system AA Dor, comparable in magnitude to the above values, cannot be considered representative of isolated stars, however. In comparison, the isolated well-studied star Feige 110 does not show obvious rotational broadening (Rauch et al. 2014), but it may be a genuine slow rotator or it could be seen under an unfavorable angle of inclination. As for the other well-studied very hot $\mathrm{H}$-sdO star, $\mathrm{BD}+28^{\circ} 4211$, the absence of detectable rotational broadening could also be due to the fact that this object may well be a post-AGB star, not a post-EHB star (Herbig 1999; Latour et al. 2013).

2 We note that the much cooler stars with $20000 \mathrm{~K} \gtrsim T_{\text {eff }} \gtrsim 11500 \mathrm{~K}$ belonging to the blue horizontal branch in globular clusters are also known to rotate slowly with values of $V_{\text {rot }} \sin i$ less than about $8 \mathrm{~km} \mathrm{~s}^{-1}$ in the sample of Behr (2003) and less than $12 \mathrm{~km} \mathrm{~s}^{-1}$ in the sample of Recio-Blanco et al. (2002).

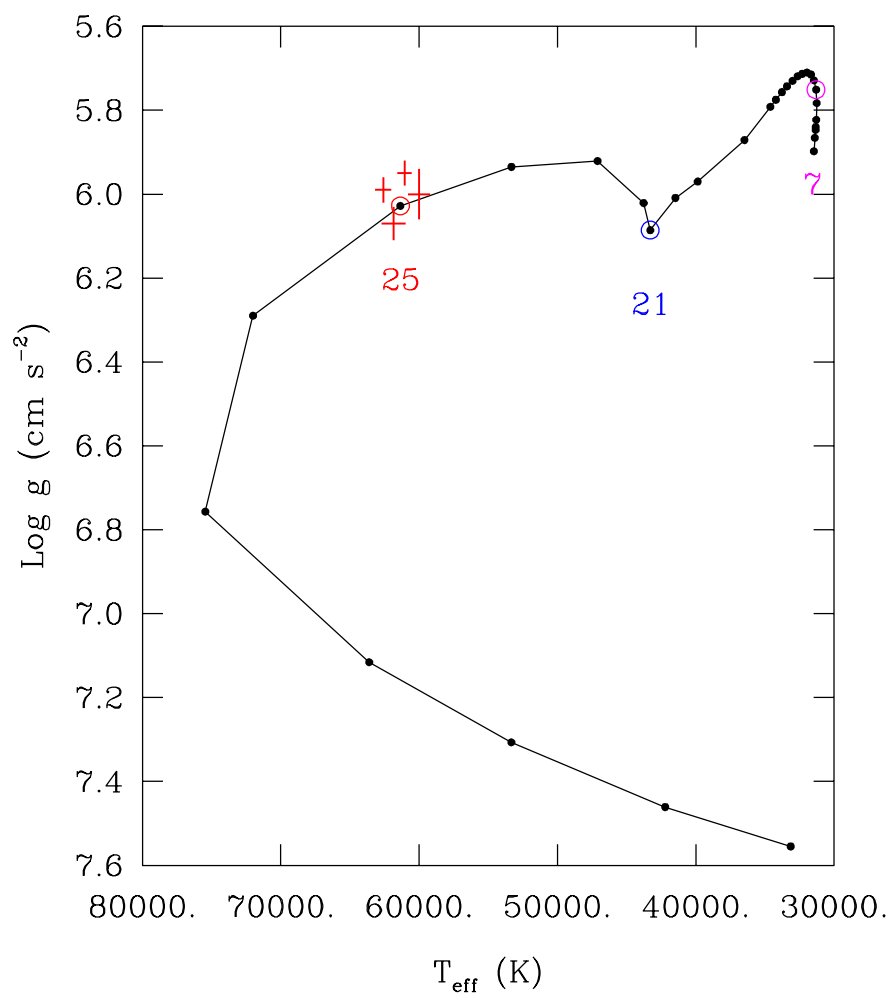

Fig. 1. Evolutionary track that passes through the region of the surface gravity-effective temperature plane where the four sdO stars of interest are located. Those are depicted by red crosses. Each available model along the track is indicated by a small black dot. Model \#7, indicated by a large open magenta circle, is representative of stars in the EHB phase of evolution and is taken as a reference. Model \#21, designated by a large open blue circle, indicates the end of the He-core burning phase. Model \#25, shown by a large open red circle, is representative of the post-EHB stars of interest.

where $M$ is the mass of the sphere. In that particular case, the ratio of the equatorial speed in the two configurations becomes,

$\frac{V_{2}}{V_{1}}=\frac{M_{1} R_{1}^{2}}{M_{2} R_{2}^{2}} \frac{R_{2}}{R_{1}}=\frac{R_{1}}{R_{2}}$,

since, by hypothesis, $M_{1}=M_{2}$, i.e., the sphere does not lose nor accrete matter. In that case, the equatorial speed scales as $V \propto 1 / R$ as mentioned above.

For the more general case of a non-uniform-density sphere, we are left with

$\frac{V_{2}}{V_{1}}=\frac{I_{1} R_{2}}{I_{2} R_{1}}$

where $I$ must be evaluated numerically according to the following relation, again available from classical mechanics,

$I=\frac{8 \pi}{3} \int_{0}^{R} \rho(r) r^{4} \mathrm{~d} r$

where $\rho$ is the density and $r$ is the radial coordinate.

We apply these principles to the case of slowly-rotating stars, i.e., with rotation slow enough for Coriolis and centrifugal forces to be negligible. As indicated above, isolated EHB stars show very little rotational broadening as a class. This means, at the very least, that their surface layers rotate very slowly indeed. Also, from asteroseismology, we know that the internal rotation 


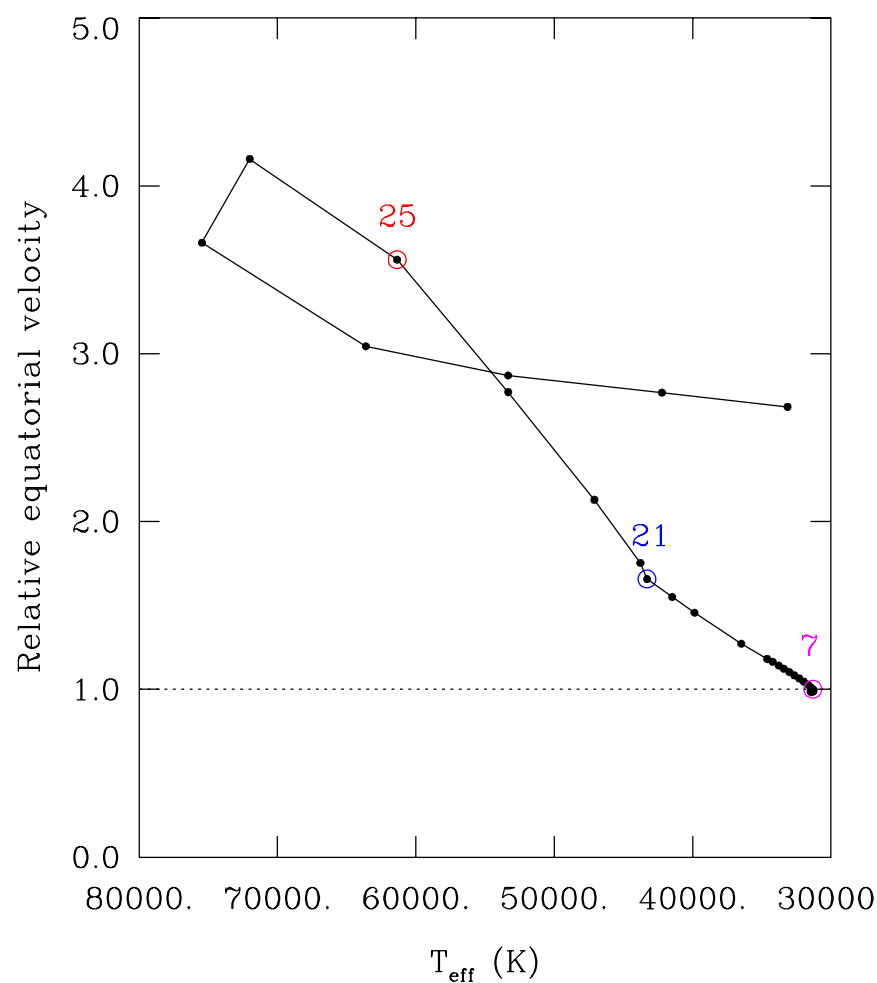

Fig. 2. Variation of the equatorial velocity along our representative track under the assumptions that angular momentum is conserved during the evolution and that the star model rotates rigidly. The format is similar to that of Fig. 1. Plotted is the ratio of the equatorial velocity with respect to that of the reference EHB model, Model \#7, as a function of effective temperature. Model \#25 is characterized by an equatorial velocity that is some 3.6 times larger than that of the reference EHB model.

profile of at least two pulsating EHB stars (Van Grootel et al. 2008; Charpinet et al. 2008) is consistent with solid-body rotation over the range of depth that can be probed with pressure modes. In the rest of this paper, and in the absence of other information, we assume that rigid-body rotation, on which is based the above equations, is a good approximation for the stars of interest.

\section{Application to evolutionary models}

Figure 1 shows the evolutionary track that we picked for our demonstration in the Kiel diagram. Each small black dot depicts one of the 31 models that are available along the track, starting with one model on the zero age extreme horizontal branch (ZAEHB) characterized by $T_{\text {eff }}=31462 \mathrm{~K}$ and $\log g=5.898$ (the dot at the beginning of the sequence in the upper right corner). This track is part of a set of seven evolutionary sequences that have been described at length in Charpinet et al. $(2000,2002)$ in their detailed study of the adiabatic pulsation properties of hot subdwarf stars. They were computed by Ben Dorman explicitly for the pulsation study of Charpinet et al., based on the same physics as that described in Dorman et al. (1993).

In their post-EHB phase, all of the seven tracks go through basically the same region where the four H-sdOs of Latour et al. (2018) are found (the four crosses in Fig. 1). The one track we picked, however, has the added quality that one of its models (Model \#25 indicated by the large open red circle) happens to fall right within the immediate region in the $\log g-T_{\text {eff }}$ diagram where the rotating $\mathrm{H}$-sdOs are found. Due to the relatively poor resolution of the sequences in the post-EHB phase, this

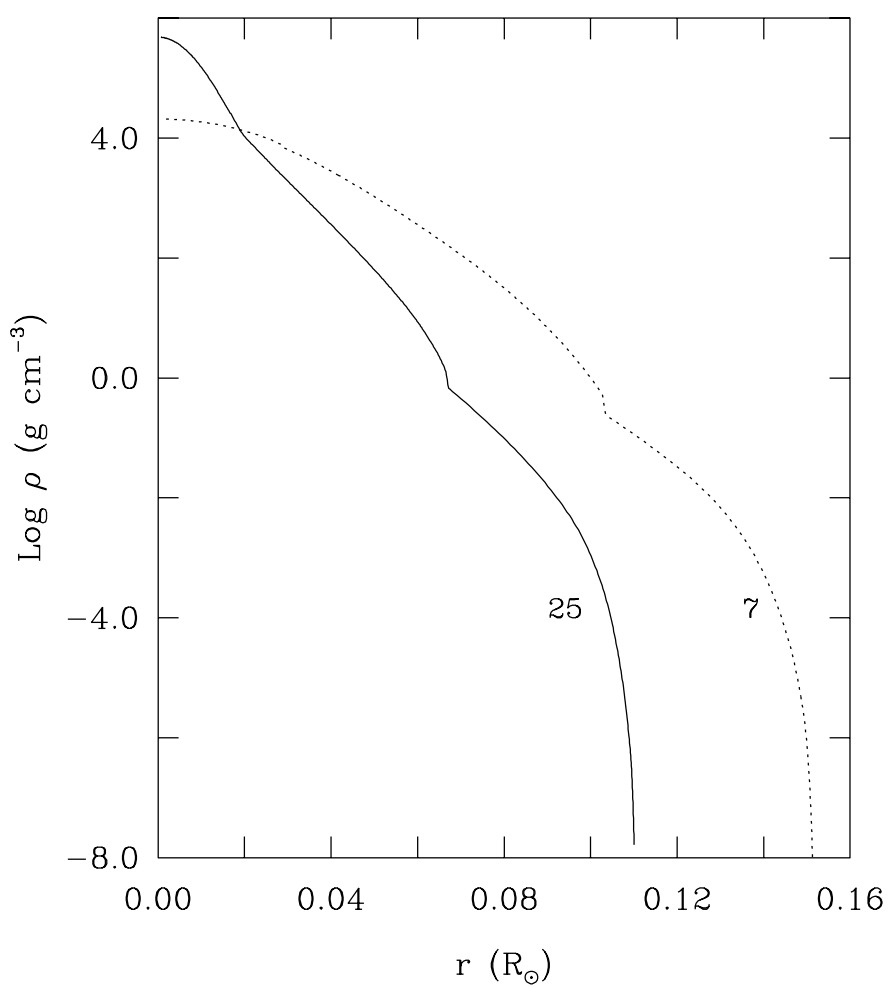

Fig. 3. Density profile in terms of the radial coordinate (in units of total solar radius) for Model \#7 (dotted curve) compared to that of Model \#25 (solid curve). The latter has a total radius some 1.4 times smaller than the former, while its central density is about 23.2 times higher. It is this central condensation of matter that is mostly responsible for the decrease of the moment of inertia of Model \#25 by a factor of about 4.9 compared to that of Model \#7.

is not the case for the other tracks, and interpolation would be needed for our comparison work. We thus adopt Model \#25 of the present sequence as our representative evolutionary model of a hot, H-rich post-EHB star.

The track illustrated in Fig. 1 belongs to a sequence defined by a core mass of $0.4758 M_{\odot}$ and an envelope mass of $0.0002 M_{\odot}$ (for a total mass of $M_{*}=0.476 M_{\odot}$ ). From this sequence, we also pick a representative model on the EHB, Model \#7, chosen somewhat arbitrarily (another choice of EHB model would have had no significant impact), and indicated by a large black circle in the plot. This EHB model is burning He in its core. A period of time of $6.19 \times 10^{7} \mathrm{yr}$ has elapsed since its beginning on the ZAEHB, and it is characterized by $T_{\text {eff }}=31310 \mathrm{~K}$ and $\log g=5.751$. For its part, Model \#25 has an age of $1.29 \times 10^{8} \mathrm{yr}$ since the ZAEHB and shines through He-shell burning (which started just after Model \#21 along the track). It is characterized by $T_{\text {eff }}=61358 \mathrm{~K}$ and $\log g=6.028$. In comparison, the average values of the atmospheric parameters of the four H-sdOs of Latour et al. (2018) are $\left\langle T_{\text {eff }}>=61400 \mathrm{~K}\right.$ and $\langle\log g\rangle=6.0$.

We computed the moment of inertia of each of the 31 retained equilibrium structures along the representative track shown in Fig. 1 with the help of Eq. (6). We next computed the ratio of equatorial speeds as given by Eq. (5) using Model \#7 as a reference. The results of these operations are presented in Fig. 2. Of central interest, the figure shows that, by the time the model has evolved from the EHB to the region of

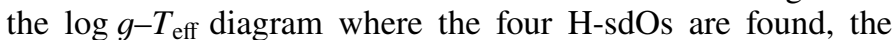
equatorial speed has increased by a factor of $\sim 3.6$. Taking the typical value of $7.7 \mathrm{~km} \mathrm{~s}^{-1}$ obtained by Geier \& Heber (2012) for isolated EHB stars, this translates into a representative value of 


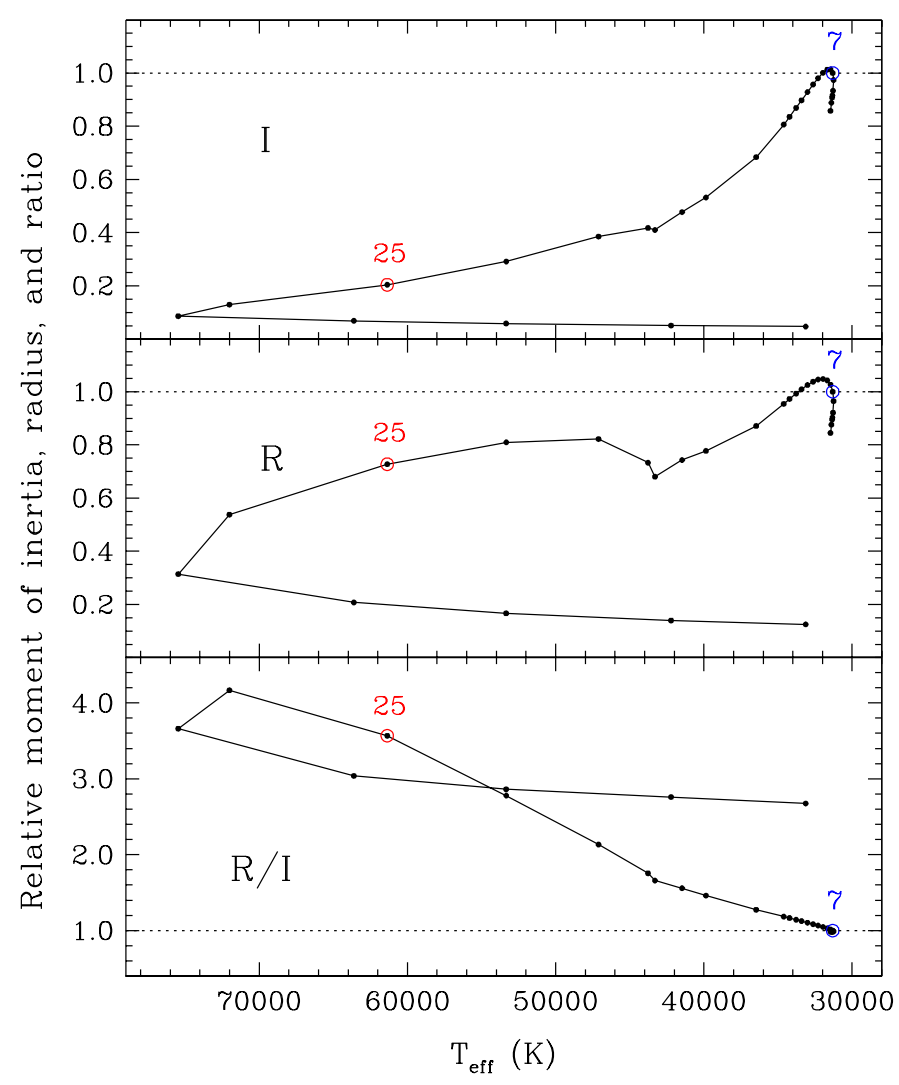

Fig. 4. Variation of the relative moment of inertia (top panel), of the relative total radius (middle panel), and of the relative ratio of the radius-to-the-moment-of-inertia (lower panel) along our representative track. The format is similar to that of Fig. 1. These quantities are plotted with respect to those of the reference EHB model, Model \#7, as a function of effective temperature. The figure reveals that the ratio of $R / I$ is maximum for Model \#26.

$V_{\text {rot }} \sin i \simeq 27.7 \mathrm{~km} \mathrm{~s}^{-1}$ for the post-EHB stars near Model \#25 in that diagram $\left(T_{\mathrm{eff}} \simeq 61400 \mathrm{~K}\right.$ and $\left.\log g \simeq 6.0\right)$. This corresponds perfectly to the values of 25 to $30 \mathrm{~km} \mathrm{~s}^{-1}$ actually inferred by Latour et al. (2018) for these stars.

To complement this discussion, we show in Fig. 3 a comparison of the density profile of Model \#25 with that of Model \#7. While the radius of the former is some 1.4 times smaller than that of the latter, its central density is some 23.2 times higher. We thus find that it is this phenomenon of central condensation produced by post-EHB evolution that is mostly responsible for the decrease of the moment of inertia and the associated spin-up of the model, not the decrease of the radius per se.

Finally, we plot in Fig. 4 the individual values of the moment of inertia $I$ (top panel), of the radius $R$ (middle panel), and of the ratio $R / I$ (lower panel) for each of the 31 models belonging to the evolutionary sequence of interest. As above, these three quantities are normalized to those of Model \#7. Given that the equatorial velocity scales as $R / I$ according to Eq. (5), and taking into account the relatively complicated behavior of both $R$ and $I$ in the figure, one can infer why the relative equatorial velocity depicted in Fig. 2 goes through a maximum near the hottest models of the sequence. Of course, the lower panel of Fig. 4 is nothing more than the equivalent of Fig. 2 above. It is of some interest to notice that on its cooling branch (models \#27 through \#31) the equatorial velocity of the resulting white dwarf decreases with passing time as its radius decreases faster than does its moment of inertia.

\section{Conclusion}

The recent work of Latour et al. (2018), combined with some previous indications, has led to the realization that isolated postEHB stars generally spin significantly faster than their immediate progenitors, the EHB stars, known to be slow rotators as a class. Using appropriate evolutionary models, we have shown that conservation of angular momentum, coupled to the assumption of rigid rotation (used as a practical working hypothesis), naturally explains the observed increase of rotational broadening by a factor of 3 to 4 from the EHB to the post-EHB phase of evolution.

Acknowledgements. The authors are grateful to the referee, Dr. Santi Cassisi, for useful suggestions. This work was supported in part by the NSERC Canada through a research grant awarded to G.F., and in part by a fellowship for postdoctoral researchers from the Alexander von Humboldt Foundation awarded to M.L. G.F. also acknowledges the contribution of the Canada Research Chair Program.

\section{References}

Becker, S. R., \& Butler, K. 1995, A\&A, 294, 215

Behr, B. B. 2003, ApJS, 149, 67

Charpinet, S., Fontaine, G., Brassard, P., \& Dorman, B. 2000, ApJS, 131, 223

Charpinet, S., Fontaine, G., Brassard, P., \& Dorman, B. 2002, ApJS, 139, 487

Charpinet, S., Van Grootel, V., Reese, D., et al. 2008, A\&A, 489, 377

Deleuil, M., \& Viton, M. 1992, A\&A, 263, 190

Dorman, B., Rood, R. T., \& O'Connell, R. W. 1993, ApJ, 419, 596

Fleig, J., Rauch, T., Werner, K., \& Kruk, J. W. 2008, A\&A, 492, 565

Fontaine, G., Green, E. M., Brassard, P., Latour, M., \& Chayer, P. 2014, in 6th

Meeting on Hot Subdwarf Stars and Related Objects, eds. V. Van Grootel,

E. M. Green, G. Fontaine, \& S. Charpinet, ASP Conf. Ser., 48183

Geier, S., \& Heber, U. 2012, A\&A, 543, A149

Han, Z., Podsiadlowski, P., Maxted, P. F. L., \& Marsh, T. R. 2003, MNRAS, 341, 669

Heber, U. 2009, ARA\&A, 47, 211

Heber, U. 2016, PASP, 128, 082001

Herbig, G. H. 1999, PASP, 111, 1144

Klepp, S., \& Rauch, T. 2011, A\&A, 531, L7

Latour, M., Fontaine, G., Chayer, P., \& Brassard, P. 2013, ApJ, 773, 84

Latour, M., Chayer, P., Green, E. M., Irrgang, A., \& Fontaine, G. 2018, A\&A, 609, A89

Németh, P., Kawka, A., \& Vennes, S. 2012, MNRAS, 427, 2180

Rauch, T., Rudkowski, A., Kampka, D., et al. 2014, A\&A, 566, A3

Recio-Blanco, A., Piotto, G., Aparicio, A., \& Renzini, A. 2002, ApJ, 572, L71

Ringat, E., \& Rauch, T. 2012, in Fifth Meeting on Hot Subdwarf Stars and Related Objects, eds. D. Kilkenny, C. S. Jeffery, \& C. Koen, ASP Conf. Ser., 452, 71

Stroeer, A., Heber, U., Lisker, T., et al. 2007, A\&A, 462, 269

Van Grootel, V., Charpinet, S., Fontaine, G., \& Brassard, P. 2008, A\&A, 483, 875

Viton, M., Burgarella, D., Cassatella, A., \& Prevot, L. 1988, A\&A, 205, 147 